\title{
Beyond dopamine: functional MRI predictors of responsiveness to cognitive behaviour therapy for psychosis
}

\section{Veena Kumari, 2*, Elena Antonova', Dominic Fannon', Emmanuelle R Peters',2, Dominic H ffytche ${ }^{3}$, Preethi Premkumar', Vinodkumar Raveendran ${ }^{1}$, Christopher Andrew ${ }^{3}$, Louise C Johns ${ }^{1}$, Philip A McGuire ${ }^{4}$, Steven CR Williams $^{3}$ and Elizabeth Kuipers ${ }^{1,2}$}

\author{
' Department of Psychology, Institute of Psychiatry, King's College London, London, UK \\ 2 National Institute for Health Research Biomedical Research Centre for Mental Health, Institute of Psychiatry and South London and Maudsley NHS Trust, London, UK \\ ${ }^{3}$ Centre for Neuroimaging Sciences, Institute of Psychiatry, King's College London, London, UK \\ ${ }_{4}^{4}$ Division of Psychological Medicine and Psychiatry, Institute of Psychiatry, King's College London, London, UK
}

\section{Edited by:}

Andreas Meyer-Lindenberg, Central Institute of Mental Health, Germany

Reviewed by:

Tilo Kircher, Universität Marburg, Germany

Bernd Gallhofer, Justus Liebig

University, Germany

${ }^{*}$ Correspondence:

Veena Kumari, Department of Psychology, PO78, Institute of Psychiatry, King's College London, De Crespigny Park, London SE5 8AF, UK. e-mail:veena.kumari@kcl.ac.uk.

\begin{abstract}
Despite the favourable effects of antipsychotics on positive symptoms of schizophrenia, many patients continue to suffer from distressing symptoms. Additional benefits of cognitive behaviour therapy for psychosis (CBTp) have been reported for approximately $50 \%$ of such patients. Given the role of left hemisphere-based language processes in responsiveness to CBT for depression, and language pathway abnormalities in psychosis, this study examined whether pre-therapy brain activity during a verbal monitoring task predicts CBTp responsiveness in schizophrenia. Fifty-two outpatients, stable on antipsychotics with at least one persistent distressing positive symptom and wishing to receive CBTp adjunctive to their treatment-as-usual, and 20 healthy participants underwent $\mathrm{fMRI}$ during monitoring of self- and externally-generated (normal and distorted) speech. Subsequently, 26 patients received CBTp for 6-8 months adjunctive to their treatment-as-usual (CBTp + TAU, 20 completers), and 26 continued with their treatment-as-usual (TAU-alone, 18 completers). Symptoms were assessed (blindly) at entry and follow-up. The $\mathrm{CBTp}+\mathrm{TAU}$ and TAU-alone groups had comparable demographic characteristics, performance and baseline symptoms. Only the CBTp + TAU group showed improved symptoms at follow-up. CBTp responsiveness was associated with (i) greater left inferior frontal gyrus (IFG) activity during accurate monitoring, especially of own voice, (ii) less inferior parietal deactivation with own, relative to others', voice, and (iii) less medial prefrontal deactivation and greater thalamic and precuneus activation during monitoring of distorted, relative to undistorted, voices. CBTp + TAU patients, on average, displayed left IFG and thalamic hypo-activation (<healthy participants). The findings implicate language processing (IFG), attention (thalamus), insight and self-awareness (medial prefrontal and parietal cortices) in CBTp responsiveness in schizophrenia.
\end{abstract}

Keywords: cognitive behaviour therapy, self, psychosis, inferior frontal gyrus, parietal lobe, verbal monitoring

\section{INTRODUCTION}

The beneficial effects of antipsychotics on positive symptoms in acutely ill patients with schizophrenia (Kasper, 2006), most likely via their actions at dopamine receptors (Kapur and Remington, 2001; Guillin et al., 2007), are well established. The long-term outcome for up to $40 \%$ of patients, however, remains unsatisfactory as they continue to suffer from one or more distressing symptoms of schizophrenia despite remaining compliant with their prescribed medication (Conley and Kelly, 2001; McEvoy et al., 2007; Potkin et al., 2009).

Kapur (2003) proposed that antipsychotics only "dampen the salience" of the abnormal experiences that cause or contribute to formation of psychotic symptoms (e.g. delusions) but do not "erase" the symptoms; symptom elimination or improvement in the longer run requires the patients to "work through" and reappraise their experiences. Embedded within the basic principles of most psychological interventions for psychiatric disorders, including cognitive behaviour therapy for psychosis (CBTp) (Fowler et al., 1995; Garety et al., 2007), is the process of working through the experiences of patients from their perspective and reappraising them (Kuipers et al., 2006; Garety et al., 2007).

Consistent with the notion of additional benefits of CBTp to pharmacotherapy alone, a large number of randomised controlled trials (RCTs) have demonstrated that persistent positive symptoms, particularly delusions, and general symptoms, such as anxiety and depression, are improved by CBTp in patients who fail to show adequate clinical response to antipsychotic therapy alone (Pilling et al., 2002; Zimmermann et al., 2005; Pfammatter et al., 2006; Wykes et al., 2008). The National Institute for Health and Clinical Excellence (NICE) updated guidelines for schizophrenia in the UK (NICE, 2009) recommend that CBTp should be offered as well as pharmacotherapy to all individuals with psychosis who request it. A meaningful clinical response to CBTp, however, is seen in only about $50 \%$ of patients who receive it (Pfammatter et al., 2006; Wykes et al., 2008). A greater understanding of the mediators of CBTp response may help to increase its benefits for the patients. 
There are few published data on predictors of response to CBTp in schizophrenia. At the neuropsychological level, cognitive flexibility is found to predict the effect of CBTp on delusional thinking (Garety et al., 1997). Clinically, cognitive insight has emerged as a potential mediator of CBTp responsiveness (Granholm et al., 2006). At the neural level, greater pre-therapy brain activity in the dorsolateral prefrontal cortex (DLPFC) and its connectivity with the cerebellum during a spatial (dot-back) working memory task has been shown to be associated with greater responsiveness to CBTp in schizophrenia, most likely via the DLPFC-cerebellum contributions to executive processing (Kumari et al., 2009). Interestingly, the association between CBTp responsiveness and greater pre-therapy DLPFC activity in this study was particularly strong for the left hemisphere, suggesting that the left-hemisphere function may be more pertinent to CBTp (Kumari et al., 2009). Greater left-hemisphere advantage for verbal processing has also been associated with a more favorable outcome of CBT for depression (Bruder et al., 1997). Given the possible link between schizophrenia and language pathway abnormalities (Crow, 2000; Li et al., 2009), the association between left-hemisphere based language processes and CBTp responsiveness may be particularly salient in this clinical population.

The present study aimed to examine the neural predictors of responsiveness to CBTp in schizophrenia using functional magnetic resonance imaging (fMRI) during a task involving monitoring of selfand externally-generated speech (Johns et al., 2001; Fu et al., 2006; Kumari et al., 2008). Accurate performance on this task produces activity changes in a neural network comprised of inferior frontal, cingulate, lateral temporal, inferior parietal, putamen and thalamic brain areas both in healthy people and patients with schizophrenia (Kumari et al., 2008). The main hypothesis, based on findings of previous studies concerning neural predictors of CBT (Bruder et al., 1997; Kumari et al., 2009), was that pre-therapy activation level of the left inferior frontal gyrus (IFG), which is known to be involved in language production (Demonet et al., 2005; Binder et al., 2009) and perception of self- and other-generated speech (Raveendran and Kumari, 2007), will be predictive of responsiveness to CBTp in schizophrenia. In addition, we expected task-related activity changes in the inferior parietal cortex since this brain region has been proposed (Shad et al., 2007) and empirically found to be associated with insight (specifically, awareness of problems) in psychosis (Cooke et al., 2008). We, however, explored task-related activations and deactivations across the entire brain as predictors of responsiveness to CBTp, given the dearth of studies on prediction of response to CBTp, as well on the brain basis of cognitive insight. Cognitive insight, unlike clinical insight, also encompasses the evaluation and correction of distorted beliefs and misinterpretations (Beck et al., 2004) and has been shown to mediate responsiveness to CBT in schizophrenia (Granholm et al., 2006). Furthermore, we also studied a group of healthy participants, matched on average to age and sex of the patient group, to investigate whether specific activity changes (if found) associated with CBTp responsiveness represented hyper-, hypo-, or normal level of activity changes.

\section{MATERIALS AND METHODS PARTICIPANTS AND DESIGN}

The study involved 56 outpatients with schizophrenia diagnosed using DSM-IV structured clinical interview (SCID) (First et al., 1995), 26 of whom received CBTp for 6-8 months in addition to their treatment-as-usual (CBTp + TAU group) while 26 continued to receive their usual treatment (TAU-alone group). A group of 20 healthy participants screened for a history of mental illness using SCID-I NP (First et al., 2002) and matched, on average, to patients on age and sex, were studied for comparison purposes. This investigation has been carried out as part of a larger project examining neural predictors and correlates of responsiveness to CBTp in schizophrenia. The sample of patients and healthy participants included in this report thus overlaps with the sample examined in our recent report (Kumari et al., 2009) on neural responsiveness of CBTp observed with fMRI of working memory (19 CBTp + TAU patients, 14 TAU-alone patients, and 15 healthy participants common to both investigations) and was included in a larger crosssectional, fMRI study of verbal monitoring (Kumari et al., 2008). Neither of these published reports investigated neural predictors of CBTp within the verbal monitoring neural network.

All participants were right-handed and had no history of neurological conditions or head injury. All included patients (i) had been on stable doses of antipsychotics for $\geq 2$ years, and on their present antipsychotic therapy for $>3$ months, (ii) received a rating of $\geq 60$ on the Positive and Negative Syndrome scale (PANSS) (Kay et al., 1987) and had at least one persistent positive symptom (a score of 3 or above on at least one of the positive symptoms items of the PANSS, which they experienced as distressing), and (iii) wished to receive 6-8 months of CBTp in addition to their usual drug treatment. Patients in both the CBTp + TAU and TAU-alone groups were recruited from the same geographical area and had been identified by their treating psychiatrists as suitable for CBTp. With the resources available at the time of this investigation to the South London and Maudsley (SLAM) NHS Foundation Trust, only about $10 \%$ of eligible patients were offered CBTp. The patients who were referred to and accepted for CBTp by the Psychological Interventions Clinic for Outpatients with Psychosis (PICuP), SLAM NHS Foundation Trust, constitute the CBTp + TAU group. The researchers did not have any say in which of the patients receive CBTp at this specialist clinic. There were no explicit biases in which patients received CBTp. This was driven by resource limitations of the NHS Trust. Others, matched to those in the CBTp + TAU group as much as possible, were allocated to the TAU-alone group. The final CBTp + TAU group had 20 patients, and the TAU-alone group had 18 patients (Table 1); these patients had remained on the same type and dosage of antipsychotic medication during the follow-up period. The main reasons for patient drop outs/exclusion from the study were consent withdrawal, medication change or non-compliance and/or acute illness exacerbation prior to follow-up.

All participants underwent fMRI during a verbal monitoring task and clinical assessment at entry. The CBTp + TAU group then received 6-8 months of CBTp following a published manual (Fowler et al., 1995) in a specialist clinical service (PICuP, South London and Maudsley NHS Foundation Trust). CBTp interventions were formulation-driven and aimed to reduce distress arising from psychotic symptoms, reduce depression, anxiety and hopelessness, and modify dysfunctional schemas when appropriate. The focus was on the therapy goals of the patient. Therapy sessions were conducted weekly or fortnightly, as preferred by the patient, and lasted for up to $1 \mathrm{~h}$. Patients received an average of 16 sessions. The therapists were qualified CBT practitioners 
Table 1 | Demographics, clinical characteristics, and task performance of participants.

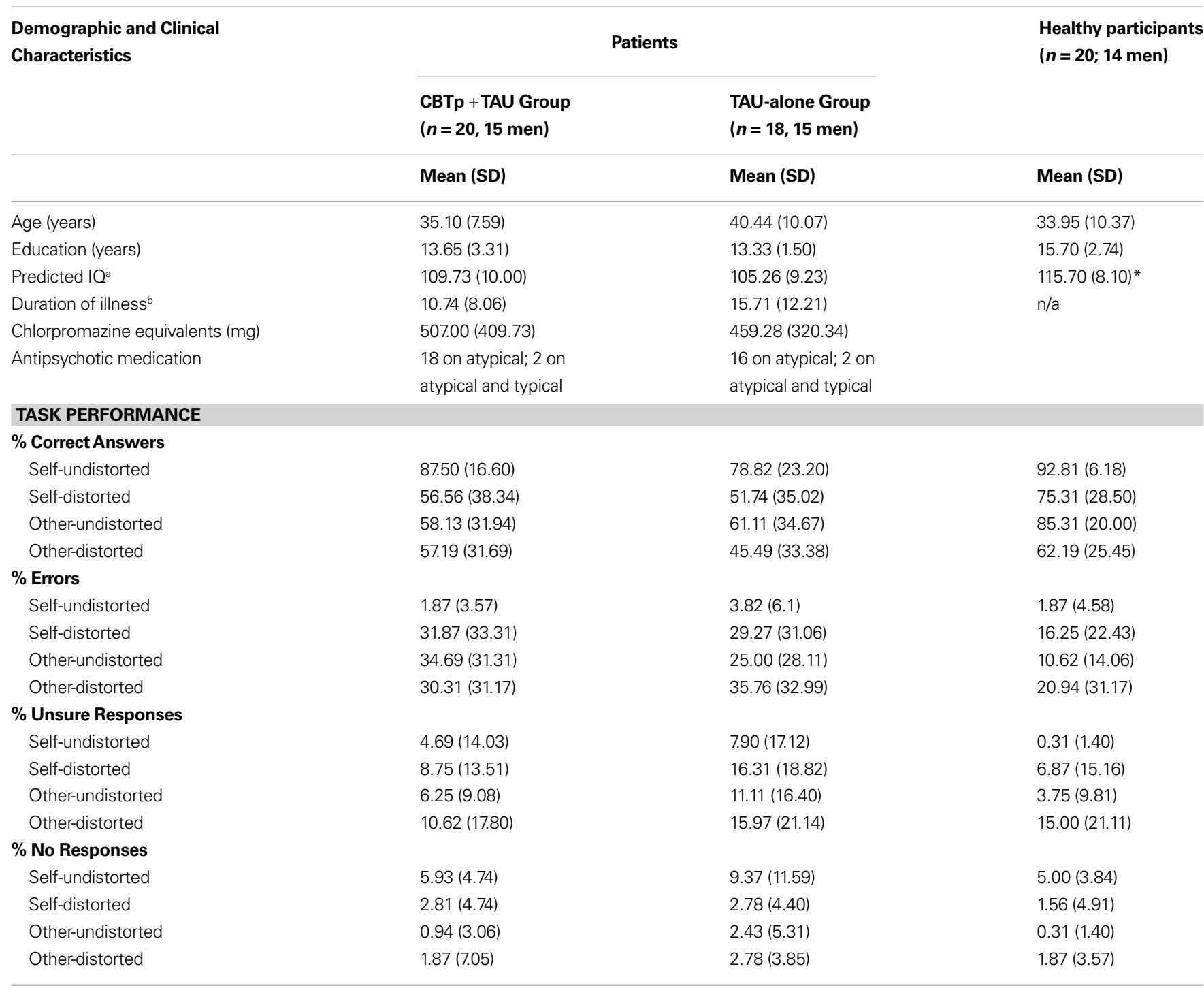

${ }^{a}$ National Adult Reading Test (Nelson and Willison, 1991); ${ }^{b}$ Duration of illness = current age minus age of illness onset; ${ }^{*} n=19$ (missing IQ data in one healthy participant).

and supervised by one of the two investigators (EK, ERP) who have extensive experience of CBTp. The treatment adherence was recorded via fortnightly supervision. In addition, a small, random selection of therapy sessions $(n=13)$ were taped and sent to an independent, experienced CBTp therapist to be rated for fidelity of treatment using the Cognitive Therapy Scale for Psychosis (Haddock et al., 2001). The mean rating was 40.7 (range 21-53) out of a maximum of 60 , with $77 \%$ of the tapes scoring above the $50 \%$ mark (i.e. $>30$ ). TAU provided to all patients prior to, and during, the study consisted of management offered by a case management team with a dedicated care-coordinator who saw the patient on a regular basis, in addition to a psychiatrist and other specialists, such as benefits adviser and vocational specialist, as needed. TAU-alone patients were followed up over the same period as CBTp + TAU patients in order to confirm CBTp led, rather than non-specific (e.g. time effect), symptom improvement in the CBTp + TAU group.
Symptoms were rated in all patients, using the PANSS (Kay et al., 1987), at entry and then 6-8 months later by an independent and experienced psychiatrist (DF) who was blind to whether or not a patient received CBTp in addition to their usual treatment. This psychiatrist had no role in recruitment and clinical management of any of the patients included in this investigation. Appointments for these assessments were made by another member of the research team.

The study procedures were approved by the joint research ethics committee of the SLAM NHS Foundation Trust and Institute of Psychiatry. After complete description of the study, written informed consent was obtained from all participants.

\section{fMRI TASK AND PROCEDURE}

A modified version (Kumari et al., 2008) of a previously described verbal monitoring task (Fu et al., 2006) was used. Participants were presented with single words visually on a computer screen 
(presentation time $750 \mathrm{~ms}$; inter-stimulus interval $16.25 \mathrm{~s}$ ), viewed via a prismatic mirror fitted in the radiofrequency head coil, as they lay in the scanner and instructed to read each word aloud. The participant's speech was transformed through a software program and a DSP.FX digital effects processor (Power Technology, California, USA), amplified by a computer sound card, and relayed back through an acoustic MRI sound system (Ward Ray-Premis, Hampton Court, UK) and pneumatic tubes within the ear protectors at a volume of $91 \mathrm{~dB}$ (SD 2). The volume of the feedback was calibrated to overcome the bone conduction of the participant's own voice. The verbal feedback was either: (a) their own voice (selfundistorted); (b) their own voice lowered in pitch by 4 semitones (self-distorted); (c) voice of another person matched to the participant's sex (other-undistorted); or (d) another person's voice with the pitch lowered by 4 semitones (other-distorted). Participants were required to register their responses regarding the origin of feedback by pressing the appropriate button on the button box provided to them using their right hand. They were instructed to press the 'self' button if they thought that the feedback was their own voice, the 'other' button if it belonged to someone else, or the 'unsure' button if they were uncertain about the nature of the feedback. On the computer screen below the words, three possible responses were written as 'self', 'other' and 'unsure' and were highlighted via a black outline every time a participant registered his/her response by pressing one of them. Accuracy of the responses was recorded online, with failures to press a response button coded as non-responses. In total, 64 words were presented (16 words per task condition, presented in a pseudo-random order).

Participants were requested to abstain from alcohol for at least $24 \mathrm{~h}$ prior to their scheduled scanning and underwent task familiarisation to familiarize them with the procedures prior to going in the scanner.

\section{IMAGE ACQUISITION}

Echoplanar MR brain images were acquired using a 1.5 T GE Signa system (General Electric, Milwaukee WI, USA) at the Maudsley Hospital, London. A quadrature birdcage head coil was used for RF transmission and reception. In each of 14 near axial non-contiguous planes (slice thickness $=7.0 \mathrm{~mm}$, interslice gap $=1 \mathrm{~mm}$ ) parallel to the inter-commissural (ac-pc) plane, T2*-weighted MR images depicting blood oxygenation level-dependent (BOLD) contrast were acquired over 1.1 s using a 'clustered' acquisition (12-14) ( $\mathrm{TE}=40 \mathrm{~ms}, 70^{\circ} \mathrm{flip}$ angle), which created a relative silent period of $2.15 \mathrm{~s}$ for each stimulus within a TR of $3.25 \mathrm{~s}$ of the inter-stimulus interval of $16.25 \mathrm{~s}$. Five brain volumes were acquired for each trial $(\mathrm{TR}=3.25 \mathrm{~s})$, and a stimulus, as stated earlier, was presented every $16.25 \mathrm{~s}$. A clustered acquisition sequence was used to reduce artefacts associated with overt speech during image acquisition (Fu et al., 2006). Foam padding within the head coil and a forehead strap were used to restrict head motion.

\section{DATA ANALYSIS}

\section{Demographic, clinical and behavioural measures}

CBTp + TAU versus TAU-alone groups: baseline comparisons. $\mathrm{CBTp}+\mathrm{TAU}$ and TAU-alone groups were compared on age, education, predicted IQ (Nelson and Willison, 1991) and baseline PANSS symptoms using independent-sample $t$-tests. Possible group differences in performance of CBTp + TAU and TAU-alone groups were examined by Group (CBTp + TAU, TAU-alone) $\times$ Source (self, other) $\times$ Distortion (undistorted, distorted) analysis of variance (ANOVA) with Group as a between-subjects factor and Source and Distortion as within-subjects factors, followed by post-hoc analyses as appropriate. Given a marked (though statistically nonsignificant) difference in age between the final CBTp + TAU and the TAU-alone groups, the effects were re-examined using analysis of co-variance (ANCOVA) with age entered as a covariate. Results only from the analysis of the correct answers are presented in detail since there were insufficient data (too few trials) to allow meaningful fMRI analysis of other performance indices (descriptive statistics for all indices presented in Table $\mathbf{1}$ ).

Effects of CBTp: symptom change in CBTp + TAU versus TAUalone groups. The change in symptoms from baseline to followup was examined using a Group $(\mathrm{CBTp}+\mathrm{TAU}$, TAU-alone $) \times$ Time (baseline, follow-up) ANOVA with Group as a between-subjects factor and Time as a within-subjects factor. Given the earlier noted difference in age between the final CBTp + TAU and the TAU-alone groups, the effects were re-examined using analysis of co-variance (ANCOVA) with age entered as a covariate. A significant Group $\times$ Time effect on total and sub-scale PANSS scores was followed up by paired $t$-tests separately in the CBTp + TAU and TAU-alone groups. Following the confirmation of significant clinical improvement in the CBTp + TAU group, and no significant change in symptoms in the TAU-alone group, potential associations between baseline symptom severity and symptom change (absolute change $=$ baseline minus follow-up) in the CBTp + TAU group were examined using Pearson's correlations. The effects of CBTp were also confirmed using ANCOVAs on symptom change scores co-varying for baseline symptoms. Potential associations between CBTp responsiveness (total PANSS scores) and baseline clinical and performance variables were examined using Pearson's correlations.

CBTp + TAUpatients(Baseline) versushealthyparticipants. We examined possible differences between the final CBTp + TAU and healthy groups in age, education and IQ using independent-sample $t$-tests, and in performance using a Diagnosis (CBTp + TAU patients; healthy participants) $\times$ Source (self, other $) \times$ Distortion (undistorted, distorted) ANOVA with Diagnosis as a betweensubjects factor and Source and Distortion as within-subjects factors, followed by post-hoc analyses as appropriate. We did not include TAU-alone patients (who a common as expected, did not show symptom improvement) in this analysis because we wished to establish performance level in the CBTp + TAU group relative to the healthy comparison group prior to examining whether the level of activity in brain areas found to associate with CBTp responsiveness reflected i.e. hyper-, hypo-, or normal level of activity.

All analyses were performed in SPSS (version 15). Prior to implementing the above described analyses, each variable was evaluated for criteria of parametric analysis. Alpha level for testing significance of effects was maintained at $p<0.05$. 


\section{fMRI}

Image pre-processing. For each participant, the functional time series were motion corrected, transformed into stereotactic space (Montreal Neurological Institute, MNI), smoothed with a $10 \mathrm{~mm}$ FWHM Gaussian filter and band pass filtered using statistical parametric mapping software (SPM2; http://www.fil.ion.ucl.ac.uk/spm).

Models and statistical inferences. Data were analysed using a random effect procedure (Friston et al., 1999). The first stage identified subject-specific activations associated with correct responding in all participants for four task conditions (self-undistorted, self-distorted, other-undistorted, other-distorted) and at the levels of Source [self-undistorted + self-distorted versus other-undistorted + otherdistorted] and Distortion (self-undistorted + other-undistorted versus self-distorted + other-distorted). Motion parameters were included as covariates at this stage. Next, we identified task-related activity changes in the CBTp + TAU group using one-sampled $t$-tests (height threshold $p<0.001$, cluster corrected $p \leq 0.05$ ).

We used the same approach as in our previous study (Kumari et al., 2009) to examine the relationship of CBTp responsiveness with pre-therapy brain activity in CBTp + TAU patients. We first computed the degree of change in symptoms independent of initial severity as residual change by regressing the initial PANSS (total and sub-scales) scores on follow-up scores as an outcome measure of CBTp responsiveness. We then regressed residual symptom change scores on task-related activity changes across the entire brain (height threshold $p=0.05$, cluster-corrected $p \leq 0.05$ ). For the positive associations of a priori hypothesised regions with CBTp responsiveness, the following significance criteria were applied to maxima voxels of clusters that did not survive whole-brain correction for multiple comparisons: (a) $T$ value of $\geq 2.88$ (corresponding to uncorrected voxel $p<0.005$ ) and $\geq 100$ contiguous voxels, and (b) survival of small volume correction (SVC) within a locally defined volume (10-mm radius sphere) with family-wise error corrected $p \leq 0.05$. Next, we extracted the subject-specific activation values from the voxels showing the strongest association with CBTp responsiveness (reduction in total PANSS symptoms) in each activity cluster and explored their possible relationships with performance and baseline symptom scores using Pearson's correlations (within SPSS).

Finally, we compared task-related activity changes in the $\mathrm{CBTp}+\mathrm{TAU}$ and healthy participant groups using independentsample $t$-tests (height threshold $p=0.05$, cluster corrected $p \leq 0.05$ ) in order to determine whether various activity changes found to associate positively with CBTp responsiveness in this patient group reflected (a) a hyper response (i.e. greater in the patient group than the healthy group), (b) a strong response within the normal range (i.e. patients not significantly different from healthy participants), or (c) a less deficient response (if, on average, this patient group showed activation deficit relative to the healthy group). We further probed patienthealthy group differences in these areas with a lenient approach using SVC within a locally defined volume $(5 \mathrm{~mm}$ radius sphere around the voxel showing the strongest association with reduction in total PANSS scores) with family-wise error corrected $p \leq 0.05$.

\section{RESULTS \\ DEMOGRAPHIC, CLINICAL AND BEHAVIOURAL MEASURES CBTp+TAU versus TAU-alone groups: baseline comparisons}

The final CBTp + TAU and TAU-alone groups were comparable in age, education, predicted IQ, illness duration, PANSS (total and subscales) symptoms and medication dose at baseline (all $p$ values $>0.05$ ). The two groups also showed comparable performance across all task conditions $\left(F_{1,36}<1\right.$ for all main and interactive effects involving Group; this remained true after co-varying for age). Both groups showed marginally higher accuracy during self than other conditions (Source: $F_{1,36}=4.19, p=0.048$ ), and markedly higher accuracy during undistorted than distorted conditions (Distortion: $F_{1,36}=46.24, p<0.001$ ), in particular during the selfundistorted condition relative to the other-distorted conditions (Source $\times$ Distortion: $F_{1,36}=4.82, p=0.035$ ).

\section{Effects of CBTp: symptom change in CBTp + TAU versus TAU-alone groups}

The CBTp + TAU group, but not the TAU-alone group, showed change in symptoms from baseline to follow-up (Group $\times$ Time, total PANSS scores: $F_{1,36}=8.59, p=0.006$; positive symptoms: $F_{1,36}=5.01, p=0.032$; negative symptoms: $F_{1,36}=5.75, p=0.022$; general psychopathology: $\left.F_{1,36}=6.31, p=0.017\right)$. The same pattern of effect was observed after we co-varied for age ( $p$ values 0.02 or higher for Group $\times$ Time interaction in the total PANSS scores and individual PANSS dimensions). Follow-up analyses revealed significant reduction in blind ratings of symptom on all dimensions (Table 2) at follow-up in only the CBTp + TAU group (total PANSS scores: $t_{19}=3.73, p=0.001$; positive symptoms: $t_{19}=3.81, p=0.001$; negative symptoms: $t_{19}=2.17$, $p=0.043$; general psychopathology: $t_{19}=3.32, p=0.004$ ).

Table 2 | Symptoms and symptom changes in the CBTp + TAU and TAU-alone patient groups.

\begin{tabular}{|c|c|c|c|c|c|c|}
\hline \multirow[t]{2}{*}{ Symptoms ${ }^{a}$} & \multicolumn{3}{|c|}{ CBTp + TAU Group Mean (SD) } & \multicolumn{3}{|c|}{ TAU-alone Group Mean (SD) } \\
\hline & Baseline & Follow-up & $\begin{array}{l}\text { Change (Baseline } \\
\text { minus Follow-up) }\end{array}$ & Baseline & Follow-up & $\begin{array}{l}\text { Change (Baseline } \\
\text { minus Follow-up) }\end{array}$ \\
\hline Positive & $18.25(4.99)$ & $\boldsymbol{\nabla} 14.90(4.30)$ & $\mathbf{\Delta} 3.35(3.94)$ & $18.72(3.12)$ & $18.28(3.56)$ & $0.44(4.06)$ \\
\hline Negative & $17.40(4.11)$ & $\boldsymbol{\nabla} 15.50(4.14)$ & $\boldsymbol{\Delta} 1.90(3.92)$ & $19.06(3.95)$ & $20.33(4.55)$ & $-1.28(4.25)$ \\
\hline General Psychopathology & $34.10(7.28)$ & $\boldsymbol{\nabla} 28.55(7.61)$ & А $5.55(7.47)$ & $34.56(4.72)$ & $34.72(6.83)$ & $-0.17(6.45)$ \\
\hline Total & $69.75(13.89)$ & $\mathbf{\nabla} 58.95$ (15.07) & $\boldsymbol{\Delta} 10.8(12.96)$ & $72.33(9.22)$ & $73.33(12.58)$ & $-1.00(11.72)$ \\
\hline
\end{tabular}

aPositive and Negative Symptom Scale (Kay et al., 1987); $\mathbf{\nabla}$ Lower symptom scores $(p<0.05)$ at follow-up in the CBTp + TAU, but not in the TAU-alone, group; $\triangle$ Symptom reduction $(p<0.05)$ in the CBTp + TAU, relative to the TAU-alone, group. 
CBTp responsiveness (total PANSS symptoms) did not significantly associate with baseline symptom severity $(r=0.37$, $p=0.107$ ). Symptom improvement (absolute change score) in the CBTp + TAU group remained significant after co-varying for baseline symptoms, relative to the TAU-alone group (total PANSS scores: $F_{1,35}=10.85, p=0.002$; positive symptoms: $F_{1,35}=7.94$, $p=0.008$; negative symptoms: $F_{1,35}=9.85, p=0.003$; general psychopathology: $\left.F_{1,35}=7.84, p=0.008\right)$. As can be expected from the independence of symptom improvement from baseline symptoms in CBTp + TAU patients, residual symptom change scores (used in fMRI analysis) correlated highly positively with the absolute symptom change scores (total PANSS symptoms: $r=0.914, p<0.001$ ). Within the CBTp + TAU group, illness duration (current age minus age at onset), education, NART IQ, antipsychotic medication dose (in chlorpromazine equivalents) and task performance (total \% correct) were not significantly associated with CBTp responsiveness $(p$ values $>0.05)$.

\section{CBTp+TAU patients (Baseline) versus healthy participants}

The final CBTp + TAU and healthy participant groups were comparable in age, but the CBTp + TAU group had marginally lower NART IQ $\left(t_{37}=2.04, p=0.05\right)$ and fewer years in education $\left(t_{37}=2.73, p=0.04\right)$ (Table 1). The patient group showed lower performance accuracy than healthy participants as indicated by a highly significant main effect of Group $\left(F_{1,38}=11.78, p=0.001\right)$. In addition, there were main effects of Source $\left(F_{1,38}=5.33, p=0.03\right.$; indicating higher accuracy for self than other conditions) and Distortion $\left(F_{1,38}=54.82, p<0.001\right.$; indicating higher accuracy for undistorted than distorted conditions), and a marginally significant Diagnosis $\times$ Source $\times$ Distortion interaction $\left(F_{1,38}=4.08, p=0.05\right)$. Further analysis of this interaction revealed that healthy participants were significantly better than CBTp + TAU patients at identifying other-undistorted voices $\left(t_{38}=3.23, p=0.003\right)$. There was a trend for better recognition of self-distorted voices in the healthy group compared to the CBTp + TAU group $\left(t_{38}=1.76, p=0.087\right)$. The two groups did not differ significantly in identifying self-undistorted or other-distorted voices $(p>0.10)$, although accuracy was somewhat lower in the patient group across all task conditions (Table 1). NART IQ or years in education did not correlate significantly with performance (total \% correct) in the CBTp + TAU group or the healthy group ( $p$ values $>0.10)$.

\section{fMRI}

\section{Generic activity changes in CBTp+TAU patients}

The generic verbal monitoring network identified revealed strongly overlapping activation and deactivation patterns for the four task conditions. This network included bilateral activations in the IFG, superior temporal gyrus, putamen, precuneus and thalamus (Table 3, Figure 1). The regions deactivated across all conditions included the middle-posterior cingulate, angular and parahippocampal gyri (Table 3, Figure 1).

\section{fMRI predictors of CBTp responsiveness}

The expected association between pre-therapy left IFG activation [Brodmann area (BA) 44-45-47] and CBTp responsiveness was found for all three PANSS symptom dimensions. This effect was more strongly and consistently present during monitoring of own undistorted speech than someone else's/own distorted speech (Table 4, Figure 2), and meant that those with a marked beneficial response to CBTp showed stronger activation of this area.

The IFG association clusters extended to the medial prefrontal cortex (BA10-BA32; located somewhat anterior and dorsal to the area deactivated across all participants) during the undistorted conditions, and were also present as higher medial prefrontal activity during undistorted compared to distorted feedback conditions. Further probing revealed that patients with the most beneficial response to CBTp did not show deactivation or showed some activation (mainly self) during the undistorted conditions, and showed deactivation of this region during the distorted conditions.

CBTp responsiveness also associated positively with (a) less deactivation/slight activation of the inferior parietal lobe (primarily BA 40 and BA7) during accurate monitoring of own, relative to someone else's, speech regardless of the level of distortion, and (b) more thalamic and precuneus activation to distorted speech relative to undistorted speech, regardless of the source (Table 4, Figure 2).

It is important to note that brain activity-CBTp response associations found in this study were present in both male and female patients (illustrated in Figure 3 with the left IGF-CBTp response association). Interestingly, pre-therapy fMRI activity and CBTp responsiveness associations were not significantly present for the other-distorted condition when this was examined as an individual task condition, perhaps because of the lowest power available (i.e. lowest number of correct answers thus less volume of fMRI data) during this condition. At the level of symptom improvement, negative symptoms dimension had the least power (smallest change with CBTp). Baseline symptoms or performance accuracy did not correlate significantly with CBTp response predictive brain regions, although a small positive association $(r=0.34, p=0.14)$ was present for the left IFG activation and accuracy during the self-undistorted condition.

\section{CBTp+TAU versus healthy participants}

CBTp + TAU patients showed significantly reduced activations, compared to healthy participants, in a number of regions during the distorted conditions, including the putamen, anterior cingulate and thalamus during the self-distorted and the left IFG during the other-distorted conditions (Table 5). Patients were also differentiated from healthy participants by reduced deactivation of parahippocampal and posterior cingulate gyri, and altered medial prefrontal cortex and caudate activity modulation between the self and other conditions.

When differences between the CBTp + TAU group and the healthy group in activations and deactivations of regions associated with CBTp responsiveness (in the CBTp + TAU group) were examined with a lenient approach (SVC), CBTp + TAU patients, relative to healthy participants, showed less activation of the left IFG $(x=-36, y=50, z=-2$; voxel $T=2.38$, uncorrected $p=0.009$, SVC-corrected $p=0.05)$. There was a trend for this effect in the thalamus $(x=6, y=-22, z=-8$; voxel $T=2.41$, uncorrected $p=0.01$, SVC-corrected $p=0.07)$. 
Table 3 | Brain areas showing significant change in task-related activity in CBTp + TAU patients (voxel threshold $p=0.001$; cluster $p$ corrected for multiple comparisons).

\begin{tabular}{|c|c|c|c|c|c|c|c|c|}
\hline \multirow[t]{2}{*}{ Brain region } & \multirow[t]{2}{*}{$\begin{array}{l}\text { Cluster size } \\
\text { (voxels) }\end{array}$} & \multirow[t]{2}{*}{ BA } & \multirow[t]{2}{*}{ Side } & \multicolumn{3}{|c|}{$\begin{array}{c}\text { MNI } \\
\text { coordinates }\end{array}$} & \multirow[t]{2}{*}{$\begin{array}{l}\text { Voxel } \\
T \text { value }\end{array}$} & \multirow[t]{2}{*}{$\begin{array}{l}\text { Cluster } \\
p \text { value }\end{array}$} \\
\hline & & & & $x$ & $Y$ & $Z$ & & \\
\hline \multicolumn{9}{|l|}{ ACTIVITY INCREASES } \\
\hline \multicolumn{9}{|l|}{ Self-undistorted } \\
\hline \multirow[t]{2}{*}{ Superior temporal gyrus } & 2597 & 22 & $\mathrm{~L}$ & -52 & -50 & 14 & 10.72 & $<0.001$ \\
\hline & & 42 & $\mathrm{~L}$ & -62 & -28 & 6 & 7.55 & \\
\hline \multirow[t]{2}{*}{ Thalamus } & 568 & $\mathrm{~N} / \mathrm{a}$ & $\mathrm{R}$ & 10 & -18 & 4 & 7.74 & $<0.001$ \\
\hline & & $\mathrm{N} / \mathrm{a}$ & $\mathrm{L}$ & -12 & -16 & 8 & 5.75 & \\
\hline Brain stem & & $\mathrm{N} / \mathrm{a}$ & $\mathrm{L}$ & -8 & -28 & -6 & 5.25 & \\
\hline Superior temporal gyrus & 3128 & 22 & $\mathrm{R}$ & 50 & -32 & 18 & 6.76 & $<0.001$ \\
\hline Lingual gyrus & & 19 & $\mathrm{R}$ & 20 & -64 & -2 & 4.18 & \\
\hline Precuneus & 270 & 18 & $L$ & -16 & -76 & 24 & 4.80 & 0.038 \\
\hline Cuneus & & 18 & $\mathrm{~L}$ & -26 & -82 & 24 & 3.80 & \\
\hline \multicolumn{9}{|l|}{ Self-distorted } \\
\hline \multirow[t]{3}{*}{ Superior temporal gyrus } & 2483 & 42 & $\mathrm{~L}$ & -60 & -24 & 16 & 11.54 & $<0.001$ \\
\hline & & 22 & $\mathrm{~L}$ & -58 & -36 & 14 & 9.09 & \\
\hline & & 42 & $\mathrm{~L}$ & -62 & -16 & 10 & 8.72 & \\
\hline Superior temporal gyrus & 3425 & 22 & $\mathrm{R}$ & 60 & -10 & 2 & 11.38 & $<0.001$ \\
\hline $\begin{array}{l}\text { (extends to the inferior } \\
\text { frontal gyrus and insula) }\end{array}$ & & 22 & $\mathrm{R}$ & 56 & -24 & 0 & 9.79 & \\
\hline Precentral gyrus & & 6 & $\mathrm{R}$ & 54 & -6 & 32 & 6.60 & \\
\hline Thalamus & & $\mathrm{N} / \mathrm{a}$ & $\mathrm{R}$ & 8 & -28 & -4 & 5.87 & \\
\hline \multirow[t]{2}{*}{ Superior temporal gyrus } & 4108 & 22 & $\mathrm{~L}$ & -50 & -30 & 12 & 9.36 & $<0.001$ \\
\hline & & 42 & $\mathrm{~L}$ & -54 & -22 & 14 & 8.59 & \\
\hline Inferior frontal gyrus & & 47 & $\mathrm{~L}$ & -40 & 22 & -6 & 6.70 & \\
\hline Superior temporal gyrus & 2650 & 22 & $\mathrm{R}$ & 54 & -22 & -2 & 8.68 & $<0.001$ \\
\hline Precentral gyrus & & 6 & $\mathrm{R}$ & 64 & -2 & 22 & 8.16 & \\
\hline Middle temporal gyrus & & 21 & $\mathrm{R}$ & 60 & -32 & 0 & 7.30 & \\
\hline \multirow[t]{2}{*}{ Cuneus } & 283 & 17 & $\mathrm{R}$ & 16 & -68 & 8 & 5.08 & 0.018 \\
\hline & & 18 & $\mathrm{R}$ & 14 & -78 & 16 & 4.81 & \\
\hline Lingual gyrus & & 18 & $\mathrm{R}$ & 14 & -82 & -2 & 4.59 & \\
\hline Other-distorted & & & & & & & & \\
\hline Thalamus & 645 & $\mathrm{~N} / \mathrm{a}$ & $\mathrm{R}$ & 12 & -16 & 4 & 7.94 & 0.001 \\
\hline & & $\mathrm{N} / \mathrm{a}$ & $\mathrm{L}$ & -10 & -22 & 6 & 7.59 & \\
\hline Brain stem & & $\mathrm{N} / \mathrm{a}$ & $\mathrm{R}$ & 10 & -28 & -2 & 5.56 & \\
\hline Superior temporal gyrus & 2490 & 42 & $\mathrm{~L}$ & -52 & -24 & 12 & 7.93 & $<0.001$ \\
\hline Precentral gyrus & & $4 / 6$ & $\mathrm{~L}$ & -52 & -14 & 42 & 6.24 & \\
\hline Superior temporal gyrus & & 22 & $\mathrm{~L}$ & -54 & -40 & 18 & 6.15 & \\
\hline
\end{tabular}


Table 3 | (Continued).

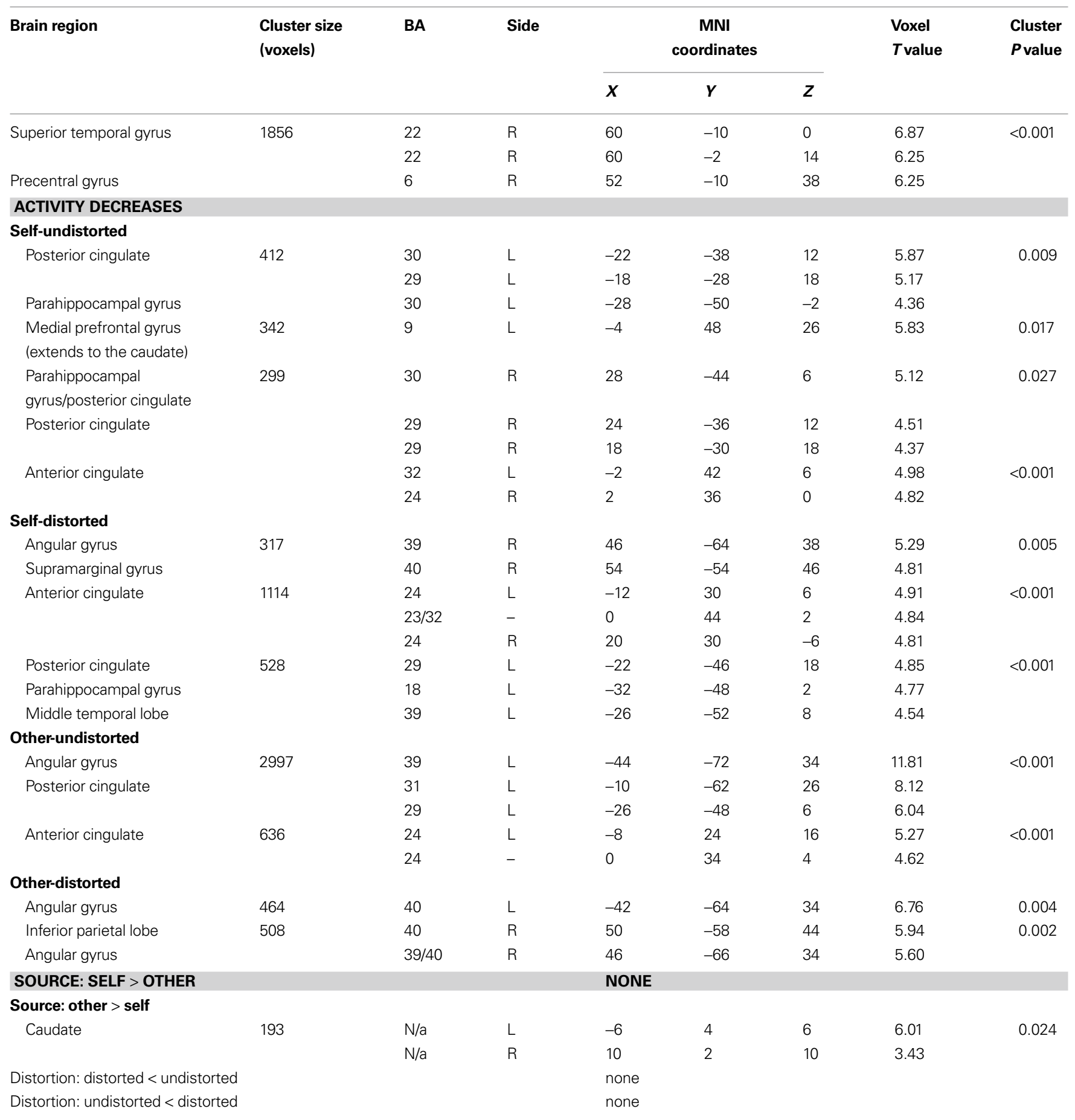

Brodmann Area $=B A ; L=$ left; $R=$ Right; self-undistorted $n=20$; Self-distorted $n=17 ;$ Other-undistorted $n=18 ;$ Other-distorted $n=18$; Self vs. Other and Undistorted $v s$. Distorted $n=15$ ( $n$ reduced due to no correct trials for few participants during a particular condition).

\section{DISCUSSION}

This investigation focussed on the association between responsiveness to 6-8 months of CBTp and pre-therapy brain activity during monitoring of self- and externally-generated speech in people with schizophrenia. It also examined a representative group of healthy participants to characterise this patient sample.
Clinically, confirming our observations in an overlapping sample (Kumari et al., 2009) and findings of recent meta analyses of RCTs for CBTp (Pilling et al., 2002; Zimmermann et al., 2005; Pfammatter et al., 2006; Wykes et al., 2008; NICE, 2009) we found reduced symptom severity, on average, in patients who received 6-8 months of CBTp adjunct to their usual treatment compared 


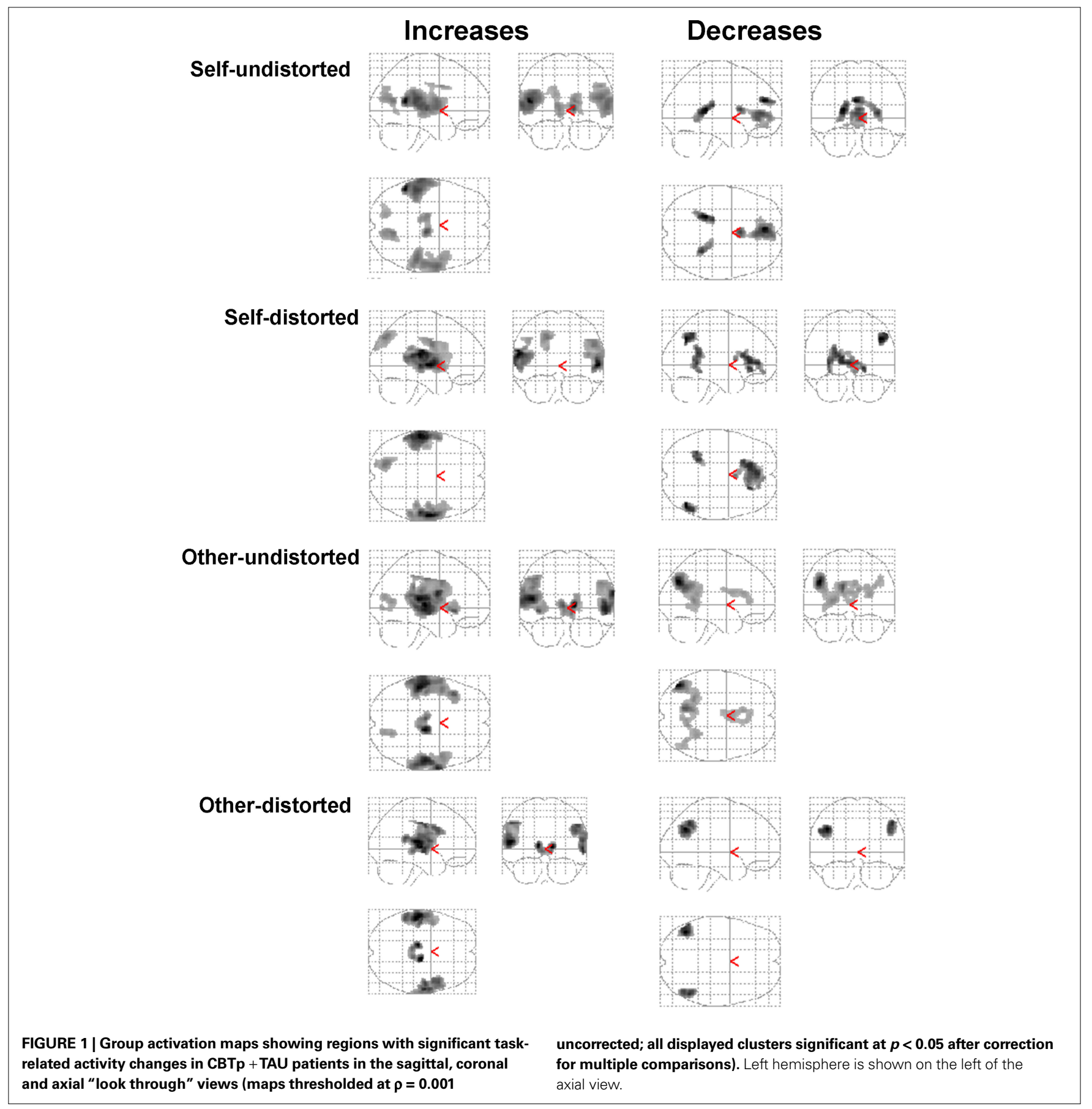

to the patient group who continued with their usual treatment. Also consistent with data from previous studies was our finding of considerable variation in the degree of symptom change for individual patients, with the change in total PANSS scores from baseline to follow-up ranging from 8 (slight worsening) to -42 (strong improvement) (Figure 3, $x$ axis).

At the neural level, as hypothesized, activation of the left IFG associated with a beneficial response to CBTp on all PANSS symptom dimensions (Table 4). This association was present more consistently during monitoring of own undistorted speech than someone else's or own distorted speech. This may at least in part be due to the fact that this condition, with the highest number of correct trials, had the maximum power to detect expected relationships. The observed left IGF-CBTp response association lends support to earlier findings of Bruder and colleagues (Bruder et al., 1997) concerning CBT for depression and can be attributed to the role of the left IFG (Broca's area) in speech and language processing (Demonet et al., 2005). The left IFG also contributes to verbal working memory (Cabeza and Nyberg, 2000; Wager and Smith, 2003) which was a likely component of performance on the task used in this study (i.e. remembering the feedback while making a decision about its origin). Given that CBTp + TAU patients also 
Table 4 | fMRI activity showing positive associations with CBTp responsiveness in patients (voxel threshold $p=0.05$ ).

\begin{tabular}{|c|c|c|c|c|c|c|c|c|}
\hline \multirow[t]{2}{*}{ Brain region } & \multirow[t]{2}{*}{$\begin{array}{l}\text { Cluster } \\
\text { size (voxels) }\end{array}$} & \multirow[t]{2}{*}{ BA } & \multirow[t]{2}{*}{ Side } & \multicolumn{3}{|c|}{$\begin{array}{c}\text { MNI } \\
\text { coordinates }\end{array}$} & \multirow[t]{2}{*}{$\begin{array}{l}\text { Voxel } \\
T\end{array}$} & \multirow[t]{2}{*}{$\begin{array}{l}p \text { value } \\
\text { (corrected) }\end{array}$} \\
\hline & & & & $x$ & $\boldsymbol{Y}$ & $z$ & & \\
\hline \multicolumn{9}{|l|}{ POSITIVE SYMPTOMS } \\
\hline \multicolumn{9}{|l|}{ Self-undistorted } \\
\hline Inferior frontal gyrus & & $45 / 46$ & $\mathrm{~L}$ & -36 & 34 & 8 & 3.15 & \\
\hline \multicolumn{9}{|l|}{ Other-undistorted } \\
\hline Medial prefrontal gyrus & 5250 & $10 / 32$ & $\mathrm{R}$ & 22 & 42 & 16 & 4.11 & 0.013 \\
\hline \multirow[t]{2}{*}{ Inferior frontal gyrus } & & 45 & $\mathrm{~L}$ & -36 & 32 & 4 & 3.58 & \\
\hline & & 45 & $\mathrm{~L}$ & -36 & 24 & 22 & 3.41 & \\
\hline \multicolumn{9}{|l|}{ NEGATIVE SYMPTOMS } \\
\hline \multicolumn{9}{|l|}{ Self-undistorted } \\
\hline Inferior-middle frontal gyrus & 3799 & $47 / 10$ & $L$ & -36 & 50 & 2 & 5.00 & 0.004 \\
\hline \multirow[t]{2}{*}{ Medial prefrontal gyrus } & & 10 & $\mathrm{R}$ & 2 & 58 & 14 & 3.92 & \\
\hline & & 10 & $\mathrm{~L}$ & -6 & 60 & 16 & 3.21 & \\
\hline \multicolumn{9}{|l|}{ Other-undistorted } \\
\hline Medial prefrontal gyrus & 1519 & 10 & $\mathrm{R}$ & 14 & 54 & -6 & 3.70 & 0.045 \\
\hline \multirow[t]{2}{*}{ Middle frontal gyrus } & & 10 & $\mathrm{R}$ & 38 & 58 & 14 & 2.68 & \\
\hline & & 10 & $\mathrm{~L}$ & -26 & 50 & -6 & 2.45 & \\
\hline \multicolumn{9}{|c|}{ GENERAL PSYCHOPATHOLOGY } \\
\hline & & 39 & $\mathrm{R}$ & 36 & -62 & 26 & 2.79 & \\
\hline Self $>$ other & & & & & & & & \\
\hline Inferior parietal lobe & 1360 & 40 & $\mathrm{~L}$ & -44 & -46 & 24 & 5.44 & 0.008 \\
\hline & & 40 & $L$ & -50 & -38 & 34 & 3.81 & \\
\hline & & 7 & $\mathrm{~L}$ & -20 & -62 & 36 & 3.56 & \\
\hline Inferior parietal lobe/ & 910 & 40 & $\mathrm{R}$ & 34 & -50 & 34 & 4.35 & 0.033 \\
\hline supramarginal gyrus & & 40 & $\mathrm{R}$ & 58 & -50 & 34 & 2.57 & \\
\hline & & 40 & $\mathrm{R}$ & 50 & -46 & 32 & 2.00 & \\
\hline Undistorted > distorted & & & & & & & & \\
\hline Middle frontal gyrus & 1727 & 10 & $\mathrm{R}$ & 42 & 48 & 4 & 4.66 & 0.020 \\
\hline Medial frontal gyrus & & $32 / 10$ & $\mathrm{R}$ & 6 & 46 & -2 & 3.85 & \\
\hline & & 10 & $L$ & -18 & 56 & -4 & 3.38 & \\
\hline TOTAL SYMPTOMS & & & & & & & & \\
\hline Self-undistorted & & & & & & & & \\
\hline Inferior frontal gyrus & 3540 & 47 & $\mathrm{~L}$ & -36 & 50 & -2 & 4.00 & 0.02 \\
\hline Medial prefrontal gyrus & & 10 & $\mathrm{~L}$ & -8 & 60 & 14 & 3.58 & \\
\hline & & 10 & $\mathrm{R}$ & 4 & 58 & 14 & 3.56 & \\
\hline
\end{tabular}


Table 4 | (Continued).

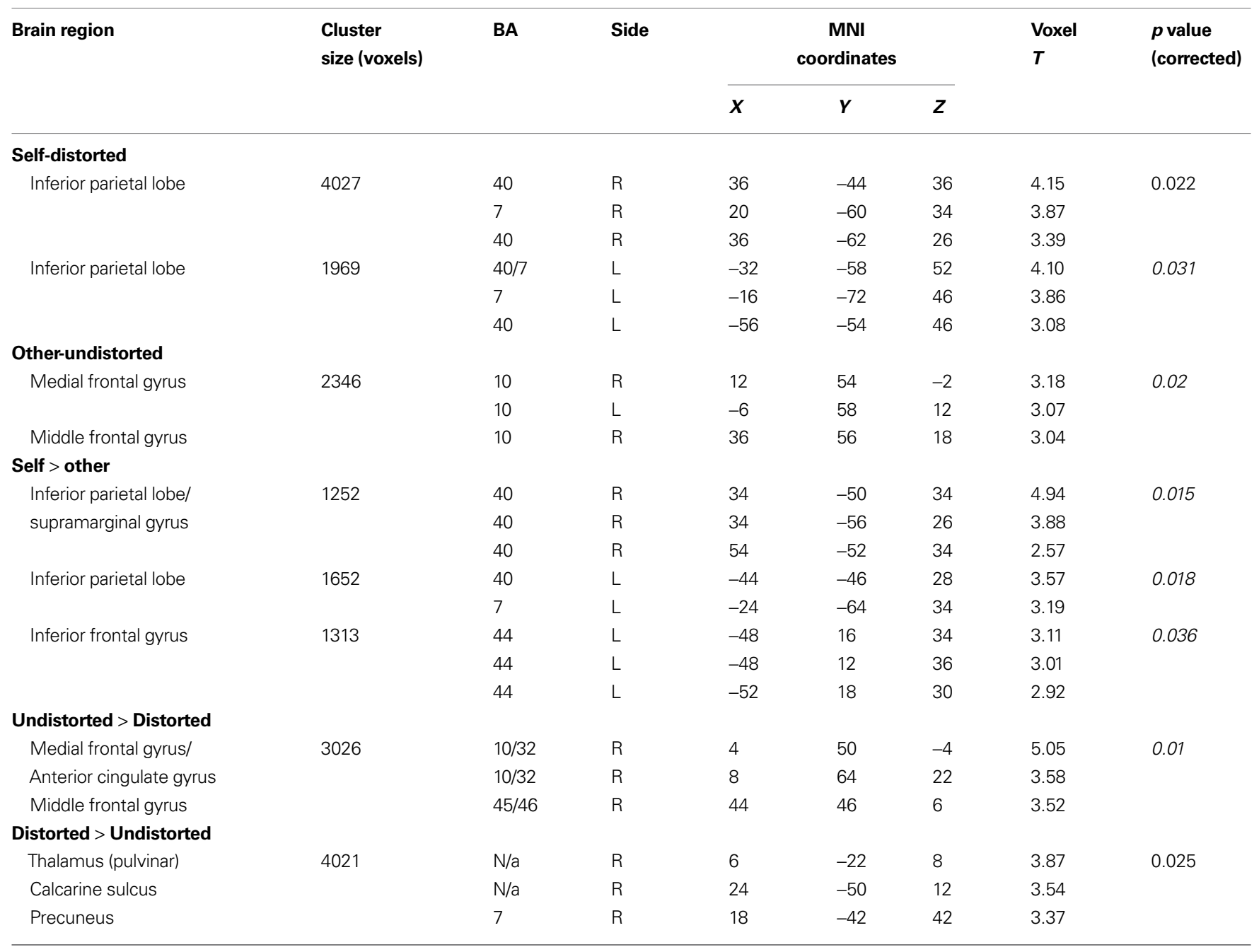

Brodmann Area=BA; L=left; R=Right. In italics: SVC criterion applied. All others: cluster p corrected for multiple comparisons across the entire brain.

displayed, on average, hypo-activation of the left IFG relative to healthy participants, our finding also suggests that normal range or less deficient activation (i.e. maximum within the patient group) boosts CBTp response. Whether such an association is specific to CBTp or also applies to other psychological interventions that involve 'talking' remain to be clarified. The lack of a significant direct association between task performance and CBTp responsiveness in this investigation may be due to the fact that task performance most likely included not only the ability to process and perceive own and someone else's speech but, in addition, other functions such as prior knowledge and experience of having used the word stimuli facilitating recognition in self-generated voice. Some components of task performance may be less relevant than others to CBTp responsiveness.

The IFG association clusters extended to the medial prefrontal cortex (BA10-BA32) during the undistorted conditions, and during undistorted compared to distorted feedback. This association showed that those with the maximum symptom improvement after CBTp mostly showed no deactivation or some activation rather than deactivation (self-undistorted) during the undistorted conditions, and showed deactivation of this region only during the distorted conditions.

Brain deactivations are commonly found during cognitive tasks (Frith et al., 1991; Binder et al., 1999; Gusnard and Raichle, 2001; Mazoyer et al., 2001; McKiernan et al., 2003, 2006; Raichle and Snyder, 2007). These are considered to reflect suspension of spontaneous thought processes, including monitoring of own body image and mental states, that occur during the rest state (default mode of brain function) while study participants are required to focus on specific external task demands (Binder et al., 1999; Gusnard and Raichle, 2001; Mazoyer et al., 2001; McKiernan et al., 2006). Although deactivations across most cognitive tasks include regions such as the posterior cingulate gyrus, dorsomedial prefrontal cortex, rostral cingulate gyrus and the angular gyrus (Binder et al., 1999; Mazoyer et al., 2001; McKiernan et al., 2003; Raichle and Snyder, 2007), the difficultly level and characteristics of the task are important determinants of the exact pattern and the extent of deactivations (McKiernan et al., 2003, 2006). More difficult levels of the 


\section{PRE-THERAPY BRAIN ACTIVITY AND RESPONSIVENESS TO CBTp}

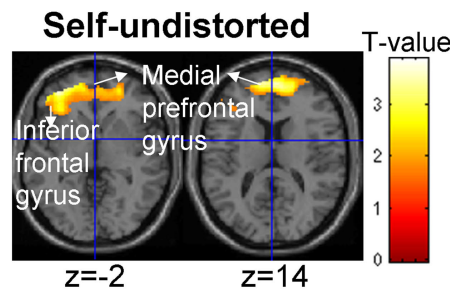

SOURCE

Self $>$ Other

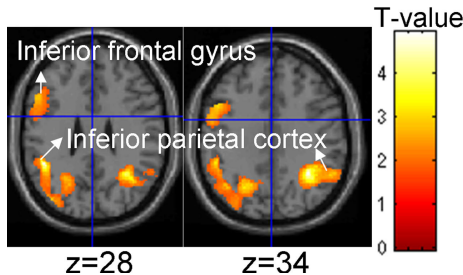

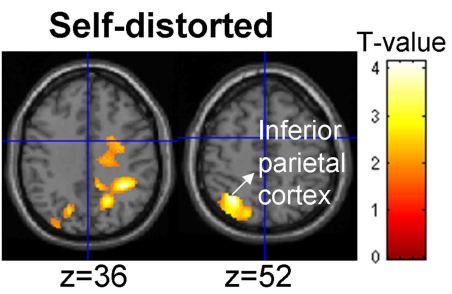

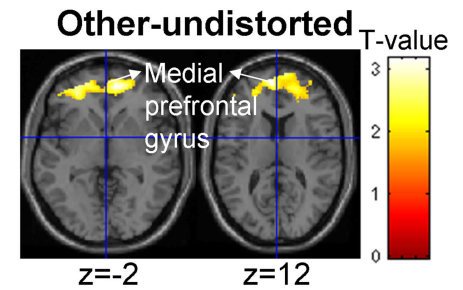

DISTORTION

Undistorted $>$ Distorted $\quad$ Distorted $>$ Undistorted

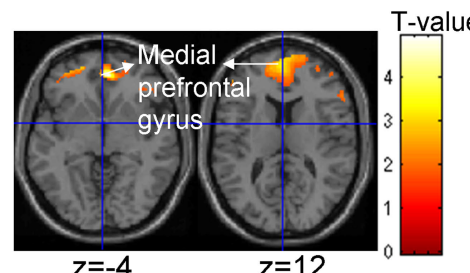

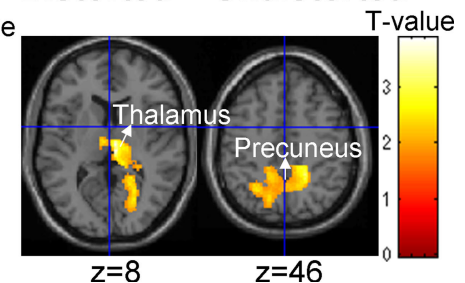

FIGURE 2 | Pre-therapy brain activity associated positively with a response to CBTp (reduction in total PANSS symptoms) in patients with associated MNI $\boldsymbol{z}$ co-ordinates (maps thresholded at $\boldsymbol{p}=\mathbf{0 . 0 5}$ uncorrected). Left hemisphere is shown on the left.

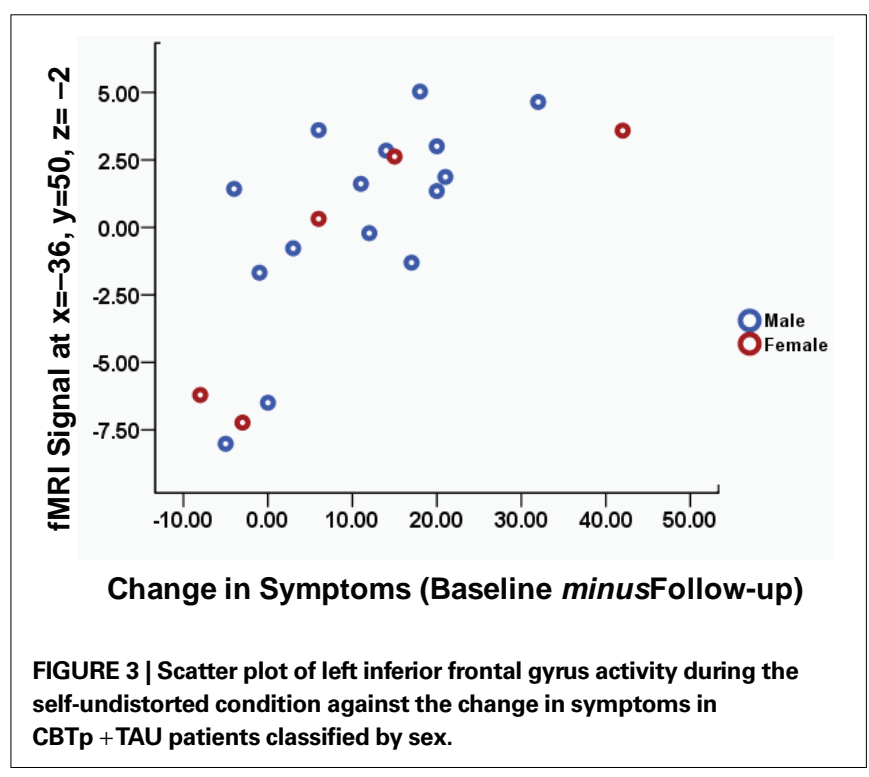

task produce larger deactivations than the easier ones (McKiernan et al., 2006), with further influence of difficulty in different domains (e.g. stimulus discriminability, working memory load) (McKiernan et al., 2003). It is obvious from our task performance that the distorted conditions were more difficult than undistorted conditions (markedly lower accuracy during the distorted relative to undistorted conditions). fMRI findings show that those failing to gain from CBTp showed maximum deactivation during the conditions of undistorted feedback (i.e. relatively easier conditions), and failed to show expected stronger deactivations with the distorted feedback conditions. Those showing a strong beneficial response to CBTp on the other hand showed marked deactivations only with the distorted conditions (i.e. the normal parametric modulation of deactivations) and showed slight (non-significant) activation during the self-undistorted condition. The latter observation is likely to be related to the processes shared by both the brain's default mode of function and the requirement of this task condition, i.e. self-monitoring (Gusnard et al., 2001; Raichle et al., 2001).

CBTp responsiveness also associated positively with less bilateral deactivation of the inferior parietal lobe (BA39-BA40-BA7) during accurate monitoring of own, relative to someone else's, voice, regardless of the level of distortion. As discussed previously (Kumari et al., 2008), there is likely to be some overlap between the 'self' conditions, which involved processing of own familiar voice, and the default baseline state (shown by the healthy group and in patients who responded well to CBTp). The findings suggest that patients benefiting most from CBTp processed self-relevant information more efficiently and displayed a greater default mode differentiation of own and others' speech.

The right parietal lobe is considered 'responsible for maintaining a self-other distinction across a variety of sensory modalities' (Uddin et al., 2005). This fits with the propositions of Shad and colleagues (Shad et al., 2007) that right inferior parietal lobe deficit contributes to anosognosia (unawareness of symptoms) via poor self-monitoring in schizophrenia. This is pertinent because cognitive insight has emerged as a mediator of response to CBT in psychotic disorders (Granholm et al., 2006). Cognitive insight in schizophrenia focuses on some of the cognitive processes (distancing, objectivity, perspective, and self-correction) involved in patients' re-evaluation of their anomalous experiences and re-interpretation of their beliefs (Beck et al., 2004) whereas clinical measurements of insight (termed 'clinical insight' by Beck and colleagues) have traditionally focused on patients' unawareness of having a mental disorder and their need for 
Table 5 | Brain areas differentiating the healthy and CBTp + TAU groups (voxel threshold $\boldsymbol{p}=\mathbf{0 . 0 5}$, cluster $\boldsymbol{p}$ corrected for multiple comparisons).

\begin{tabular}{|c|c|c|c|c|c|c|c|c|c|}
\hline \multirow[t]{2}{*}{ Brain Region } & \multirow[t]{2}{*}{$\begin{array}{l}\text { Cluster } \\
\text { size (voxels) }\end{array}$} & \multirow[t]{2}{*}{ BA } & \multirow[t]{2}{*}{ Side } & \multicolumn{3}{|c|}{$\begin{array}{c}\text { MNI } \\
\text { Coordinates }\end{array}$} & \multirow[t]{2}{*}{$\begin{array}{l}\text { Voxel } \\
T\end{array}$} & \multirow[t]{2}{*}{$\begin{array}{l}\text { Cluster } \\
p\end{array}$} & \multirow[t]{2}{*}{$\begin{array}{l}\text { Direction } \\
\text { of effect }\end{array}$} \\
\hline & & & & $\boldsymbol{x}$ & $y$ & $z$ & & & \\
\hline \multicolumn{10}{|l|}{ SELF-UNDISTORTED } \\
\hline \multicolumn{10}{|c|}{ No significant difference between healthy participants and patients } \\
\hline \multicolumn{10}{|l|}{ SELF-DISTORTED } \\
\hline Thalamus (pulvinar) & & $\mathrm{n} / \mathrm{a}$ & $\mathrm{R}$ & 30 & -26 & 6 & 2.87 & & $\begin{array}{l}\text { Greater activation in healthy } \\
\text { participants relative to patients }\end{array}$ \\
\hline \multicolumn{10}{|l|}{ OTHER-UNDISTORTED } \\
\hline \multicolumn{10}{|c|}{ No significant difference between healthy participants and patients } \\
\hline \multicolumn{10}{|l|}{ OTHER-DISTORTED } \\
\hline Inferior frontal gyrus & 10760 & 47 & $\mathrm{~L}$ & -42 & 16 & -6 & 5.31 & $<0.001$ & $\begin{array}{l}\text { Greater activation in healthy } \\
\text { participants relative to patients }\end{array}$ \\
\hline Posterior cingulate gyrus & \multirow{2}{*}{5051} & 29 & $\mathrm{~L}$ & -14 & -36 & 10 & 3.71 & \multirow{2}{*}{0.023} & \multirow{2}{*}{$\begin{array}{l}\text { Less deactivation in patients } \\
\text { relative to healthy participants }\end{array}$} \\
\hline Lingual gyrus & & 19 & $\mathrm{R}$ & 16 & -66 & 0 & 3.10 & & \\
\hline \multicolumn{10}{|c|}{ SOURCE: SELF VS. OTHER } \\
\hline Medial frontal gyrus & 4459 & 10 & $\mathrm{R}$ & 20 & 50 & 2 & 3.24 & 0.018 & $\begin{array}{l}\text { Greater medial prefrontal } \\
\text { gyrus deactivation during } \\
\text { other, relative to self in healthy } \\
\text { participants; opposite effect } \\
\text { in patients }\end{array}$ \\
\hline \multirow[t]{2}{*}{ Caudate nucleus } & & $\mathrm{n} / \mathrm{a}$ & $\mathrm{L}$ & -8 & 10 & 4 & 3.21 & & \multirow{2}{*}{$\begin{array}{l}\text { Caudate deactivation to self, } \\
\text { and slight activation to other, } \\
\text { conditions in patients but not } \\
\text { in healthy participants }\end{array}$} \\
\hline & & $\mathrm{n} / \mathrm{a}$ & $\mathrm{R}$ & 14 & 2 & 10 & 3.15 & & \\
\hline
\end{tabular}

\section{DISTORTION: UNDISTORTED VS. DISTORTED}

No significant difference between healthy participants and patients

Brodmann Area $=B A ; L=$ left; $R=$ Right

treatment, usually focussed on their need for medication. Cognitive and clinical insight measures are reported to be moderately correlated in psychotic individuals (Pedrelli et al., 2004). Although the neural substrates of cognitive insight in schizophrenia have yet not been investigated, they may at least partially overlap with those areas reported or proposed for clinical insight (Shad et al., 2007) and also involve the right parietal lobe. It should, however, be noted that CBTp response in our study was associated with activity changes in both the left and the right inferior parietal lobes. While a strong case has already been made by previous researchers for the right parietal lobe involvement in self-monitoring and insight in schizophrenia (Shad et al., 2007), our ability to infer someone else's perspective is reported to depend on functions of the left temporo-parietal junction (Samson et al., 2004, 2005). It is conceivable that CBTp (and good cognitive insight) would be facilitated when a patient is able to infer someone else's (e.g. therapist's) perspective and generate an alternative perspective to interpret his/her abnormal experiences.

Greater thalamic and precuneus activation to distorted, relative to undistorted, speech regardless of the source (self, other) also associated with a greater response to CBTp.
Stronger activation of the thalamus during distorted versus undistorted feedback might indicate increased attention to distorted (more difficult) speech stimuli (Adler et al., 2001) in CBTp responders. Given that $\mathrm{CBTp}+\mathrm{TAU}$ patients also displayed, on average, a trend for thalamic hypo-activation relative to healthy participants, this finding may suggest greater CBTp led benefits in those with relatively normal thalamic activation or a smaller activation deficit. In healthy volunteers, precuneus activation has been found in association with awareness of the self (Andreasen et al., 1995), comparing self to non-self representations (Kircher et al., 2000, 2002), and reflecting about own personality traits and physical appearance (Kjaer et al., 2002). In patients with schizophrenia, larger precuneus volume is associated with good insight, especially the awareness of problems (Cooke et al., 2008). Increased precuneus activation during distorted conditions thus could be associated with CBTpP responsiveness via increased awareness of own mental states.

It is important to highlight that activity changes found to be associated with CBTp responsiveness in this study are different to those that were associated with specific symptom profiles (i.e. 
exaggerated middle-superior temporal activations with positive symptoms, ventral striatal and hypothalamic activity alterations with negative symptoms) in our earlier study (Kumari et al., 2008), which included patients from this study.

This investigation had limitations. Firstly, it did not use a random design, due to resource limitations, for allocation of patients to CBTp + TAU and TAU-alone groups. The patients, however, were randomly distributed across the two groups in terms of their desire to receive CBTp. Furthermore, the aim of this study was to investigate neural predictors of CBTp in patients who undergo this therapy (in addition to their usual treatment) and the primary analyses to achieve this aim utilized the data obtained only in the CBTp + TAU group. Secondly, the final CBTp + TAU and TAU-alone groups differed slightly (non-significantly) in age and illness duration. This happened because of unbalanced drop outs in the two patient groups and was beyond our control. It however may not affect our results in terms of neural predictors since age or illness duration was not a significant predictor of CBT response in our sample. Thirdly, it can be argued that CBTp + TAU patients showed symptom improvement simply because of therapist contact, independent of the specific effects of the CBT methods applied to them. This is, however, unlikely. A large number of RCTs have shown that CBTp has specific effects on symptoms, as opposed to other psychological interventions such as family therapy (decreases relapse and hospitalization rates), social skill training (helps acquire social skills) or cognitive remediation therapy (improves cognitive functioning) (Zimmermann et al., 2005; Pfammatter et al., 2006; Wykes et al., 2008), and is superior in reducing the symptoms to a non-specific befriending intervention controlling for the amount of contact with treating professionals (Sensky et al., 2000). Finally, given that some regions found to be associated with CBTp responsiveness did not show robust task-related activity changes at the

\section{REFERENCES}

Adler, C. M., Sax, K. W., Holland, S. K., Schmithorst, V., Rosenberg, L., and Strakowski, S. M. (2001). Changes in neuronal activation with increasing attention demand in healthy volunteers: an fMRI study. Synapse 42, 266-272.

Andreasen, N. C., O'Leary, D. S., Cizadlo, T., Arndt, S., Rezai, K., Watkins, G. L., Ponto, L. L., and Hichwa, R. D. (1995). Remembering the past: two facets of episodic memory explored with positron emission tomography. Am. J. Psychiatry 152, 1576-1585.

Beck, A. T., Baruch, E., Balter, J. M., Steer, R. A., and Warman, D. M. (2004). A new instrument for measuring insight: the Beck Cognitive Insight Scale. Schizophr. Res. 68, 319-329.

Binder, J. R., Desai, R. H., Graves, W. W., and Conant, L. L. (2009). Where is the semantic system? A critical review and meta-analysis of 120 functional neuroimaging studies. Cereb. Cortex 19, 2767-2796.

Binder, J. R., Frost, J. A., Hammeke, T. A., Bellgowan, P. S., Rao, S. M., and Cox, R. W. (1999). Conceptual processing

group level (though present when examined at a relatively lenient threshold; data not shown), further studies using tasks which more robustly activate these brain regions (for example, for the left IFG, the use of a verbal fluency paradigm requiring generation of words as opposed to the current paradigm which required participants to only read the words that were presented to them on the screen) are needed to confirm our findings.

\section{CONCLUSIONS}

The findings demonstrate positive associations between CBTp responsiveness in schizophrenia and greater left IFG activity during accurate monitoring, especially of own voice, less inferior parietal deactivation/slight activation with own, relative to someone else's, voice, and less medial prefrontal deactivation and greater thalamic and precuneus activation with monitoring of distorted, relative to undistorted, voices. Given the known functions of these regions in humans, the findings implicate language processing (left IFG), attention (thalamus), and insight and self-awareness (medial prefrontal, inferior parietal, precuneus) in responsiveness to CBTp in schizophrenia, and suggest avenues for future development/modification of CBTp for patients who appear relatively less responsive to current versions of the routine CBTp. Targeting insight and self-monitoring skills very early during the course of CBTp may help to improve response rates. CBTp may also need to be modulated further for patients with impaired language processing skills. Clinically, given scarce resources, it may be helpful to prioritise those with good working memory and unimpaired language skills, when offering CBTp.

\section{ACKNOWLEDGMENTS}

The study was supported by funds from the Wellcome Trust, UK (067427/z/02; Senior Research Fellowship to Veena Kumari).

Demonet, J. F., Thierry, G., and Cardebat, D. (2005). Renewal of the neurophysiology of language: functional neuroimaging. Physiol. Rev. 85, 49-95.

First, M. B., Spitzer, R. L., Gibbon, M., and Williams, J. B. W. (1995). Structured Clinical Interview for DSM-IV Axis I disorders, Patient Edition (SCID-P), version 2. New York, NY, New York State Psychiatric Institute, Biometrics Research.

First, M. B., Spitzer, R. L., Gibbon, M., and Williams, J. B. W. (2002). Structured Clinical Interview for DSM-IV-TR Axis I Disorders, Research Version, Non-patient Edition. (SCID-I/NP). New York, NY, New York State Psychiatric Institute, Biometrics Research.

Fowler, D., Garety, P. A., and Kuipers, E. (1995). Cognitive Behaviour Therapy for Psychosis: Theory and Practice. Chichester, Wiley.

Friston, K. J., Holmes, A. P., and Worsley, K. J. (1999). How many subjects constitute a study? Neuroimage 10, 1-5.

Frith, C. D., Friston, K. J., Liddle, P. F., and Frackowiak, R. S. (1991). A PET study of word finding. Neuropsychologia 29, 1137-1148.

Fu, C. H., Vythelingum, G. N., Brammer, M. J., Williams, S. C., Amaro, E., Jr. Andrew, C. M., Yaguez, L., van Haren, N. E., Matsumoto, K., and McGuire, P. K. (2006). An fMRI study of verbal self-monitoring: neural correlates of auditory verbal feedback. Cereb. Cortex 16, 969-977.

Garety, P., Fowler, D., Kuipers, E., Freeman, D., Dunn, G., Bebbington, P., Hadley, C., and Jones, S. (1997) London-East Anglia randomised controlled trial of cognitive-behavioural therapy for psychosis. II: Predictors of outcome. Br. J. Psychiatry 171, 420-426.

Garety, P. A., Bebbington, P., Fowler, D., Freeman, D., and Kuipers, E. (2007) Implications for neurobiological research of cognitive models of psychosis: a theoretical paper. Psychol. Med.37, 1377-1391.

Granholm, E., Auslander, L. A., Gottleib, J. D., McQuaid, J. R., and McClure, F. (2006). Therapeutic factors contributing to change is cognitive-behavioural 
group therapy for older persons with schizophrenia.J. Contemp. Psychother. 36, 31-41.

Guillin, O.,Abi-Dargham,A., and Laruelle, M. (2007). Neurobiology of dopamine in schizophrenia. Int. Rev. Neurobiol. 78, 1-39.

Gusnard, D. A., Akbudak, E., Shulman, G. L., and Raichle, M. E. (2001). Medial prefrontal cortex and self-referential mental activity: relation to a default mode of brain function. Proc. Natl. Acad. Sci. U.S.A. 98, 4259-4264.

Gusnard, D. A., and Raichle, M.E. (2001). Searching for a baseline: functional imaging and the resting human brain. Nat. Rev. Neurosci. 2, 685-694.

Haddock, G., Devane, S., Bradshaw, T., McGovern, J., Tarrier, N., Kinderman, P., Baguley, I., Lancashire, S., and Harris, N. (2001). An investigation into the psychometric properties of the cognitive therapy scale for psychosis (CTS-Psy). Behav. Cogn. Psychother. 29, 221-233.

Johns, L.C., Rossell, S., Frith, C., Ahmad,F., Hemsley, D., Kuipers, E., and McGuire, P. K. (2001). Verbal self-monitoring and auditory verbal hallucinations in patients with schizophrenia. Psychol. Med. 31, 705-715.

Kapur, S. (2003). Psychosis as a state of aberrant salience: a framework linking biology, phenomenology, and pharmacology in schizophrenia. Am. J. Psychiatry 160, 13-23.

Kapur, S., and Remington, G. (2001). Dopamine $\mathrm{D}(2)$ receptors and their role in atypical antipsychotic action: still necessary and may even be sufficient. Biol. Psychiatry 50, 873-883.

Kasper, S. (2006). Optimisation of longterm treatment in schizophrenia: treating the true spectrum of symptoms. Eur. Neuropsychopharmacol. 16(Suppl. 3), S135-S141.

Kay, S. R., Fiszbein, A., and Opler, L. A. (1987). The positive and negative syndrome scale (PANSS) for schizophrenia. Schizophr. Bull. 13, 261-276.

Kircher, T. T., Brammer, M., Bullmore, E., Simmons, A., Bartels, M., and David, A. S. (2002). The neural correlates of intentional and incidental self processing. Neuropsychologia 40, 683-692.

Kircher, T. T., Senior, C., Phillips, M. L., Benson, P. J., Bullmore, E. T., Brammer, M., Simmons, A., Williams, S. C., Bartels, M., and David, A. S. (2000). Towards a functional neuroanatomy of self processing: effects of faces and words. Brain Res. Cogn. Brain Res 10, 133-144.
Kjaer, T. W., Nowak, M., and Lou, H. C. (2002). Reflective self-awareness and conscious states: PET evidence for a common midline parietofrontal core. Neuroimage 17, 1080-1086.

Kuipers, E., Garety, P., Fowler,D., Freeman, D., Dunn, G., and Bebbington, P. (2006). Cognitive, emotional, and social processes in psychosis: refining cognitive behavioral therapy for persistent positive symptoms. Schizophr. Bull. 32(Suppl. 1), S24-S31.

Kumari, V., Fannon, D., Ffytche, D. H., Raveendran, V., Antonova, E., Premkumar,P.,Cooke,M.A.,Anilkumar, A. P., Williams, S. C., Andrew, C., Johns, L. C., Fu, C. H., McGuire, P. K., and Kuipers, E. (2008). Functional MRI of verbal self-monitoring in schizophrenia: performance and illness-specific effects. Schizophr. Bull. [Epub ahead of print].

Kumari, V., Peters, E. R., Fannon, D., Antonova, E., Premkumar, P., Anilkumar, A. P., Williams, S. C., and Kuipers, E. (2009). Dorsolateral prefrontal cortex activity predicts responsiveness to cognitive-behavioral therapy in schizophrenia. Biol. Psychiatry 66, 594-602.

Li, X., Branch, C. A., and DeLisi, L. E. (2009). Language pathway abnormalities in schizophrenia: a review of fMRI and other imaging studies. Curr. Opin. Psychiatry 22, 131-139.

Mazoyer, B., Zago, L., Mellet, E., Bricogne, S., Etard, O., Houde, O., Crivello, F., Joliot, M., Petit, L., and TzourioMazoyer,N. (2001). Cortical networks for working memory and executive functions sustain the conscious resting state in man. Brain Res. Bull. 54, 287-298.

McEvoy, J. P., Lieberman, J. A., Perkins, D. O., Hamer, R. M., Gu, H., Lazarus, A., Sweitzer, D., Olexy, C., Weiden, P., and Strakowski, S. D. (2007). Efficacy and tolerability of olanzapine, quetiapine, and risperidone in the treatment of early psychosis: a randomized, doubleblind 52-week comparison. Am. J. Psychiatry 164, 1050-1060.

McKiernan, K. A., D’Angelo, B. R., Kaufman, J. N., and Binder, J. R. (2006). Interrupting the "stream of consciousness": an fMRI investigation. Neuroimage 29, 1185-1191.

McKiernan, K.A., Kaufman, J.N., KuceraThompson, J., and Binder, J. R. (2003). A parametric manipulation of factors affecting task-induced deactivation in functional neuroimaging. J. Cogn. Neurosci. 15, 394-408.
Nelson, H., and Willison, J. (1991). National Adult Reading Test Manual. Windsor, Nfer-Nelson.

NICE. (2009).National InstituteforClinical Excellence in the United Kingdom: Guidelines for Schizophrenia. London, Gaskell Press.

Pedrelli, P., McQuaid, J. R., Granholm, E., Patterson, T. L., McClure, F., Beck, A. T., and Jeste, D. V. (2004). Measuring cognitive insight in middle-aged and older patients with psychotic disorders. Schizophr. Res. 71, 297-305.

Pfammatter, M., Junghan, U. M., and Brenner, H. D. (2006). Efficacy of psychological therapy in schizophrenia: conclusions from metaanalyses. Schizophr. Bull. 32(Suppl. 1), S64-S80.

Pilling, S., Bebbington, P., Kuipers, E., Garety, P., Geddes, J., Orbach, G., and Morgan, C. (2002). Psychological treatments in schizophrenia: I. Metaanalysis of family intervention and cognitive behaviour therapy. Psychol. Med. 32, 763-782.

Potkin, S. G., Weiden, P. J., Loebel, A. D., Warrington, L. E., Watsky, E. J., and Siu, C. O. (2009). Remission in schizophrenia: 196-week, double-blind treatment with ziprasidone vs. haloperidol. Int. J. Neuropsychopharmacol. 12, 1-16.

Raichle, M. E., MacLeod, A. M., Snyder,A. Z., Powers, W. J., Gusnard, D. A., and Shulman, G.L. (2001). A default mode of brain function. Proc. Natl. Acad. Sci. U.S.A. 98, 676-682.

Raichle, M. E., and Snyder, A. Z. (2007). A default mode of brain function: a brief history of an evolving idea. Neuroimage 37, 1083-1090; discussion 1097-1089.

Raveendran, V., and Kumari, V. (2007). Clinical, cognitive and neural correlates of self-monitoring deficits in schizophrenia: an update. Acta Neuropsychiatrica 19, 27-37.

Samson, D., Apperly, I. A., Chiavarino, C. and Humphreys, G. W. (2004). Left temporoparietal junction is necessary for representing someone else's belief. Nat. Neurosci. 7, 499-500.

Samson, D., Apperly, I. A., Kathirgamanathan, U., and Humphreys, G. W. (2005). Seeing it my way: a case of a selective deficit in inhibiting self-perspective. Brain 128, 1102-1111.

Sensky, T., Turkington, D., Kingdon, D., Scott, J. L., Scott, J., Siddle, R., O'Carroll, M., and Barnes, T.R. (2000). A randomized controlled trial of cognitive-behavioral therapy for persistent symptoms in schizophrenia resistant to medication. Arch. Gen. Psychiatry 57, 165-172.

Shad, M. U., Keshavan, M. S., Tamminga, C. A., Cullum, C. M., and David, A. (2007). Neurobiological underpinnings of insight deficits in schizophrenia. Int. Rev. Psychiatry 19, 437-446.

Uddin, L. Q., Kaplan, J. T., MolnarSzakacs, I., Zaidel, E., and Iacoboni, M. (2005). Self-face recognition activates a frontoparietal "mirror" network in the right hemisphere: an event-related fMRI study. Neuroimage 25, 926-935.

Wager, T. D., and Smith, E. E. (2003). Neuroimaging studies of working memory: a meta-analysis. Cogn. Affect Behav. Neurosci. 3, 255-274.

Wykes, T., Steel, C., Everitt, B., and Tarrier N. (2008). Cognitive behavior therapy for schizophrenia: effect sizes, clinical models, and methodological rigor. Schizophr. Bull. 34, 523-537.

Zimmermann, G., Favrod, J., Trieu, V. H., and Pomini, V. (2005). The effect of cognitive behavioral treatment on the positive symptoms of schizophrenia spectrum disorders: a meta-analysis. Schizophr. Res. 77, 1-9.

Conflict of Interest Statement: The authors declare that the research was conducted in the absence of any commercial or financial relationships that could be construed as a potential conflict of interest.

Received: 12 August 2009; paper pending published: 30 November 2009; accepted: 18 January 2010; published online: 12 February 2010.

Citation: Kumari V, Antonova E, Fannon $D$, Peters ER, ffytche DH, Premkumar $P$, Raveendran $V$, Andrew C, Johns LC, McGuire PA, Williams SCR, and Kuipers E (2010) Beyond dopamine: functional MRI predictors of responsiveness to cognitive behaviour therapy for psychosis. Front. Behav. Neurosci. 4:4. doi: 10.3389/neuro.08.004.2010

Copyright (c) 2010 Kumari, Antonova, Fannon, Peters, ffytche, Premkumar, Raveendran, Andrew, Johns, McGuire, Williams, and Kuipers. This is an openaccess article subject to an exclusive license agreement between the authors and the Frontiers Research Foundation, which permits unrestricted use, distribution, and reproduction in any medium, provided the original authors and source are credited. 